\title{
Communication in the Sciences as Seen through Physics and Chemistry: A Look at the Complex Relationship between Author, Publisher, and Distributor as They Relate to the Reader
}

\section{William W. Armstrong}

\begin{abstract}
Writing has long been the primary means of communicating in the sciences, yet the nature of the written word is rapidly changing as we enter a new era of electronic communications and virtual realities. This article examines some of these changes, particularly as they pertain to the disciplines of chemistry and physics and, most important, within the scope of the complex relationship between authors, publishers, and distributors (distributors in this case being academic libraries). This examination involves looking at changes within this triumvirate, the relationship each of the three has with the other, and ramifications of the changes as we peer into the near future. The three members of the triumvirate are intricately and inextricably bound together, and problems that occur within any one component will inevitably affect the others, imperiling the relationship between writer and reader. Such potential problems are brought to light in this article.
\end{abstract}

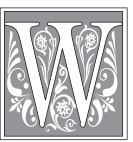

riting has long been the primary means of communicating in the sciences, a means of transmitting and disseminating new discoveries that, in turn, provide ideas for further research and other discoveries. Indeed, the written word has long held primacy in the academic world of ideas, yet the nature of the written word is rapidly changing as we enter a new era of electronic communications and virtual realities.
The concept of writing itself is entering this new "virtual" domain, where its existence is dependent on that tenuous transmission of bits and bytes that somehow continually reform themselves into the thoughts we had originally expressed when entering the codes that form the necessary characters for the transmission of meaning. Yes, we still print these characters out and read them as we have in some form or another since the fourth millennium BCE. But that is

William W. Armstrong is Head of the Chemistry Library at Louisiana State University; e-mail: notwwa@lsu. edu. 
changing, as is the way in which we actually write and communicate. And the capabilities of the electronic medium with its rapidly evolving software dedicated to writing are fueling the change, along with the advancement of the means of distributing our writings.

This article looks at some of these changes, particularly as they pertain to the disciplines of chemistry and physics and, most important, within the scope of the complex relationship between authors, publishers, and distributors (distributors in this case being academic libraries). This exploration involves looking at changes within the triumvirate of authors, publishers, and distributors, the relationship that each has with the other, and the ramifications of those changes as we look into the near future. The triumvirate members are intricately and inextricably bound together, and problems that occur within any one will inevitably affect the others. It is precisely such potential problems that this article will bring to light.

This article first looks at the nature of writing itself in two model disciplines-physics and chemistry-to see how authors in these fields best communicate. How does the ideal means of communication for those authors affect their relationships with their publishers and distributors? And, in turn, how do changes made within publishers and distributors affect the authors and, most important, the constituent for whom all three groups exist-the reader? Then the article looks for pitfalls around the corner and uncovers whatever may lie ahead for the writer. These concerns will most certainly mirror problems that other writers in other disciplines will have to face sooner or later and within the same bound relationship with publishers and distributors that the focus writers in this article experience.

\section{Communication within Physics and Chemistry}

How and what do physicists communicate? (And the "what" really determines the "how.") Physicists study the fundamental forces of the universe. One might say that they communicate abstract ideas concerning the causes of things, not unlike philosophers, but largely through mathematical equations. As with mathematicians, the world for them is often expressed in this means. Consequently, the ability to transmit ideas involves the ability to use mathematical symbols, so the methods by which this is and can be done electronically must be explored.

The nature of the world for chemists, however, is quite different. Chemistry is the study of the properties, composition, and transformation of matter. It is a world explored and seen at the molecular level, with all the various interactions that can occur between molecules. To put it very simply, chemists play with molecules - all kinds of molecules. It is a hands-on world, by and large, not an abstract one. Chemists transform matter. They have to see the world and feel it in order to understand it. The visual aspect, then, is essential to chemistry in understanding the makeup of a compound or the reaction one compound may have with another. But how does one visualize at the molecular level? By what means do chemists communicate to each other discoveries or revelations in the molecular world? This discussion will show that the traditional word is often not as important as the image. And how the image can now be produced and reproduced has changed dramatically in the digital world of writing. Indeed, with both physicists and chemists, words as a primal means of communicating have been replaced by images or symbols. And with the extremely rapid advances in electronic communications today, it is as though the art-and even the nature-of 
writing has taken a quantum leap backward and forward at the same time, backward to a pseudo-hieroglyphic system and forward to a means of production and transmission that offers possibilities never before available to the scientist. But is there the potential for disaster if one is unaware of the rapidly shifting nature of the path one treads? This is primarily what must be examined - the nature of the ground upon which we walk in this new electronic medium.

\section{Physics: Authors and Publishers}

Looking at writing within physics, with both authors and publishers of physics journals, by what means do physicists translate their thoughts into communicable symbols? One program favored by many physicists and also used by the American Physical Society (APS) and the American Institute of Physics (AIP) for author submission of papers is called LaTeX, created by Leslie Lamport in $1985 .{ }^{1}$ LaTeX is basically a typesetting program that uses the TeX engine designed by Donald E. Knuth, Professor Emeritus at Stanford. It is a macro compiler that processes specific macro sets such as those provided by the LaTeX program. In combination, these two allow authors to create high-quality output of content containing mathematical and technical expressions. LaTeX features a number of predefined sets, or templates, of various formatting styles within which one can write, including book, article, report, sectioning capabilities, cross-referencing, indexing, and even bibliography creation. The inclusion of such commands within the structure allows the author to concentrate solely on content, without having to worry about how the document is going to look. The only drawback is that the program lacks the user-friendly nature of one such as Microsoft (MS) Word or Corel Word Perfect, arguably the two most popular and widely accepted commercial word-processing programs. LaTeX also has somewhat of a learning curve; the user must gain a certain command of the language used. As might be expected, this lack of a user-friendly nature has produced a demand from more and more authors for programs that are easier to use, particularly as the commercial ones become better at dealing with and creating symbols, pictures, and technical expressions. Even the creator of LaTeX is inclined to agree. Asked what features he would like to add to the program, Lamport replied, "I would like to see a system that combines the good features of Word, such as ease of use for doing simple things, with the good features of LaTeX, such as the relative ease of separating logical content from formatting. I have ideas on how to design such a system, but it would take several people years to do, so it probably won't happen."2 But once learned, the author can basically type away, unconcerned about the output, because that will take care of itself based on the template used, not automatically possible in MS Word or Word Perfect.

By and large, LaTeX fills the need many physicists have for a program when writing, but another component to physics communication is equally important and becoming more problematic as it becomes increasingly prevalent. That component is the use and necessity of high-quality images in the transmission of content and information. This has ramifications within the entire communication process between author and reader that manifest themselves primarily at the publisher and distributor level. These problems are discussed later in this article when their effect is examined at the publisher/distributor level.

The needs or demands of the author greatly affect the publisher and how the publisher goes about its business. But so, 
too, do publisher demands have a great effect on the nature and style of the authors' writing. So a program used by an author must be accepted by the publisher and, conversely, programs insisted on or mandated by publishers must be taken into account and used by the authors when writing and submitting articles. LaTeX has been introduced as one of the more prevalent programs used by physicists, but it is important to take a look at this issue from the publishers' side to see what programs and formats they will accept for articles submitted for publication.

Two publishers in physics are the APS and the AIP mentioned earlier. APS offers its own version of LaTeX called REVteX, which provides templates with the macros necessary for outputting in the standard layout and style of all APS journals. Further encouragement has been given to authors using this by allowing publication charge discounts for all manuscripts submitted in the REVteX format. ${ }^{3}$ A fairly recent development (July 2002), however, was the acceptance of submissions in MS Word format, though only for Physical Review Letters and Physical Review Special Topics-Accelerators and Beams, both of which also receive the page charge discount. As of March 2003, APS extended the acceptance of Word to the Physical Review A-E journals. As one might expect, many authors have been demanding acceptance of MS Word by APS because of its user-friendly nature, and APS has been obliging, although REVTeX remains its preferred format for submission of electronic texts. But there are compatibility issues between proprietary software designed for the end user, such as MS Word, which might be used by an author, and nonproprietary software, which runs on UNIX systems used by some publishers in preparing manuscripts for publication. As APS stated on its FAQ (frequently asked questions) page, "One of the fundamental difficulties is that Microsoft does not supply a version of Word that will function on any UNIX system, and none of the supposed alternatives for handling MS Word files actually do a very good job with the proprietary Microsoft formats. We've tried Adobe's Word for Word, Word Perfect, Sun's Star Office, and the Keypak conversion utilities; each had serious problems with line lengths and spacing, footnotes, or particularly graphical/OLE inclusions in the text. Our entreaties to Microsoft to provide a UNIX version of Word that actually works have received no response." 4

So publisher needs will continue to have a great impact on what the author may or may not do, despite what he or she wishes. Although, of course, those very wishes, when strong enough, drive the publishers in their decisions. But more important, what begins to manifest itself already in these examinations is the very serious universal problem that lies ahead-that of software compatibility.

What about the actual process of manuscript submission? This, too, is part of the entire process of communicating and is undergoing enormous changes both in and because of the digital environment, changes that affect each member of the triumvirate identified earlier. APS has an online, Web-based submission process whereby an author can submit a paper via special programs provided in the online submission site. Included in the process is the ability to select the correct journal, the number and type of files, and the e-print number and the ability to identify and upload files, add or delete any files, and describe the files. Also, the author may complete any manuscript/journal details and provide color figure authorization and copyright transfer, among other things. Should any changes be necessary, from either the author's or the editor's 
standpoint, a resubmission process also is provided. The entire process in either of the submission scenarios remains digital, though the peer-review process is not accounted for within the scope of this service. ${ }^{5}$

Through its online hosting platform, Scitation (formerly Online Journal Publishing Service, or OJPS), AIP also endeavors to provide as complete a digital environment as possible that will allow for maximum advantage and use of new technological capabilities. It offers a new Web-based peer-review and editorial management service called Peer X-Press, which brings authors, reviewers, editors, and staff together for the task of processing new manuscripts more efficiently on the way to publication. Offered in this service are online manuscript submission and viewing, Web access to pertinent editorial process-tracking data, electronic tools to conduct the peer-review process, editorial management reports, and direct transfer to AIP production. ${ }^{6}$

AIP also features composition services for authors, allowing them to supply manuscripts via disk, e-mail, or FTP and in a variety of formats. AIP will supply author toolkits, or templates, for preparing manuscripts in REVTeX, MS Word, and Word Perfect, as well as guidelines for the preparation of graphic files. Apparently, AIP's staff has developed means for translating the most popular of the commercial formats, MS Word and Word Perfect, as well as the capability of handling REVTeX files. The author has maximum flexibility for creating in whatever environment suits him or her best. File formats acceptable for graphics include PostScript, EPS, or TIFF files; original artwork, photos, or transparencies can be scanned. However, specific application files such as those from Corel Draw cannot be accepted. AIP will provide conversion programs for producing JPEG and GIF files, if needed for
Web delivery. In addition to the aforementioned services, AIP provides thorough SGML tagging to enable extensive linking to other articles and databases, a feature of electronic writing that is becoming ever more prevalent and in demand.

What is developing between writer and publisher is a very complex relationship regarding writing, communication, and the handling of manuscripts involving a continuous evolution of programs, something to keep in mind as this article progresses.

\section{Chemistry: Authors and Publishers}

Chemistry presents a very special aspect of writing because of the nature of the field, for the language of chemistry is really that of structures, not words, and revolves around the structural formulas of molecules and compounds. Chemical information is best communicated-and often best searched for-via structural drawings. ${ }^{7}$ Physical structures represent by and large the most informative way to understand the nature of a compound. Those that can be represented in 3D provide an even better mechanism for understanding. This, of course, goes back to the discussion of chemistry as a handson field where spatial understandings go hand-in-hand with spatial representations, and the latter seem critical to the understanding of chemistry itself.

In an article entitled "Structural Chemistry and Spatial Ability of Different Cultures," Hans-Dieter Barke and Temechegn Engida point out the close connection between spatial ability and the ability to understand chemical structures. ${ }^{8}$ Emphasizing the importance of images and imagination in this process, the authors quote Einstein, who stated that "Imagination is more important than knowledge." It is literally through the imagination, or images, that we truly begin to develop an understanding of any given matter (in the 
very literal sense, in this case) at hand. What is its molecular and physical structure? This helps lead to understanding how it might behave or react in different circumstances, based on both its structure and properties.

Of course, as with all models of understanding, the spatial, visual representation can perhaps lead to problems when confronted with "real" existence. A case in point is the controversy within chemistry concerning orbitals. ${ }^{10}$ Eric R. Scerri recently stated the problem nicely in an editorial in Foundations of Chemistry. "Whereas theoreticians regard these entities as being mere mathematical constructs, devoid of any physical significance, people in all other areas of chemistry as well as chemical education tend to adopt an approach which favors the visualization of orbitals. As a result of such visualization, which is of course one of the main ways that chemists operate successfully, the physical existence of orbitals is sometimes wrongly entertained in that the model is confused with reality." 11 This remark was prompted by a claim made by J. M. Zuo and colleagues published in Nature (1999) to the effect that they had indeed directly observed orbitals, a claim given further credence by Colin J. Humphreys in the News and Views section of the same issue, where he flatly stated: "The classic textbook shape of electron orbitals has now been directly observed. As well as confirming the established theory, this work may be a first step to understanding high-temperature superconductivity." 12,13

The point here is not to go into the ramifications to the foundation of chemistry that the truth or falsity of such a claim may entail but, rather, to illustrate the importance of, and reliance on, images and imagination by chemists in understanding the molecular world in which they work. And this is of direct relevance to the topic at hand-writing and the manner in which images fit into the way chemists write, transmit information, and even look for information.

The images that are most important here are, of course, 3D images that, in the physical world, can be provided via physical models. But what about the traditionally flat world of writing and printing? Of course, we are stuck with two-dimensional representations in print, but the migration to a digital format and means of communicating has opened up possibilities previously unavailable to chemists, students, and researchers alike. But are these means available in the still rather traditional form of the scholarly journal? If not, what are the barriers to such use? And, if so, might problems ensue from the unexamined use of such possibilities?

Before examining these possibilities open to the field of chemistry, one must first take a look at the current reality in the writing and transmission, publication, and dissemination of scholarly journal articles in chemistry. Chemistry researchers, of course, are still dependent on the traditional written word despite the importance of visible chemical structures in communicating meaning. The American Chemical Society (ACS), one of the major publishers of chemistry research, accepts several different formats for hard-copy submissions, including Microsoft Word, Word Perfect, and restricted use of LaTeX. ${ }^{14}$ In addition, ACS provides templates for each of its journals in several versions of MS Word and Word Perfect, the exact format and version depending on the nature of the submission (for example, whether it is a communication or an article).

But ACS is not restricted to these software types when it comes to accepting manuscripts and the submission process 
itself. It has recently developed a new Web-based online manuscript submission and processing system called the Paragon System. Paragon was gradually phased into operation, journal by journal, until virtually all ACS journals are now able to use it. The system supports a few extra types of software unavailable for use by the hard-copy submitter, including PDF, other versions of LaTeX, and Postscript. ${ }^{15}$

ACS also provides detailed information on the preparation of graphics, as these play a large role in the information conveyed within the prospective article. Specifications for chemical structures and for text in figures and graphs are designed to ensure that the figure or graphic will reproduce sufficiently well so that no information is lost to the reader. Photographs also must adhere to certain restrictions and guidelines.

To use Paragon, the prospective author must register with the system, thereby creating a profile for him- or herself. After reviewing the available author documentation for the system, the author must download and use the appropriate manuscript template provided by ACS, which is journal specific. Finally, the appropriate copyright and copyright permission forms must be downloaded. The system allows authors or their designees to upload all pertinent files, including manuscript and supporting information, to the specific journal to which the manuscript is being submitted. The author can subsequently sign in at any time to check the status of the submission. Likewise, reviewers can register in the system and obtain copies of the articles they will review through the Paragon site.

Again, it is clear that as things become easier for authors in processes such as writing and article submission, they simultaneously become more complex in the layers beneath the surface.

\section{Distributors: Libraries}

The third component of the triumvirate described in this article comprises the distributors, or libraries. This component encompasses the distribution group within academia, an essential element in bringing the final written product to its readership, without which, of course, writing itself makes little sense.

So how does the entire process of writing and publication within the disciplines of this article's focus affect libraries' handling of the written word $?^{16}$ And what light might the nature of this third component shed on problematic issues facing the writer within the electronic medium? An examination of this final member of the triumvirate is necessary to bring into focus crucial, but hidden, issues to the writer.

First, what is the library's role in the entire process after the "word" has been written and published? Basically, the library's task is threefold: to organize the information (assuming the ability to purchase, of course), to provide access, and to preserve. All of these tasks are essential to the prospective reader's ability to obtain and read an article, and remain conceptually the same as they have always been; however, the method of fulfilling these tasks has changed dramatically in the digital environment. To organize a journal collection, for example, a library constructs electronic records for titles the library "owns" and organizes them within the scope of an electronic catalog system that provides a detailed bibliographic record of a given journal. This organization consists less of providing call numbers to the journals than of providing indexing enabling a patron to find the item via a journal title search, a subject search, or a keyword search. And, as with print versions, the organization of electronic titles is directly connected to access, although an additional step is 
needed with e-journals. Whereas with print an assigned call number suffices to enable a patron to find a journal on the shelves of a library, a hyperlink is required to send the user to the location of the e-journal. This method of access is radically different from the method used for the corresponding print version of a journal, as the Web-based journals and content can be provided to faculty at their desktop.

The above paragraph implies a potential problem with the term own or ownership, and this concept is intimately tied to the third role of the library as distributor-preservation. What role will this facet of libraries play in the relationship among writers, publishers, and libraries as they negotiate the new electronic waters? And this role will be crucial in evaluating the actions of the other two and, ultimately, in determining a course of action and plotting a path through this as-yet-unexplored territory. For at stake here is the concept of maintaining a permanent relationship between writer and reader over time.

In this realm of ownership can be detected a marked and revolutionary change that electronic writing and publication has brought about. The question of ownership is far more tenuous in the electronic world of publication than in the print world. With print, a library subscribes to a journal and, on a regular basis, receives physical copies of volumes and issues that belong to the library as long as it wishes to keep them. Implicit in this arrangement is the responsibility of preservation of these volumes, which has fallen among the library's responsibilities from the early days of publication. But this is rapidly changing, solely because of the nature of electronic publishing as it is emerging today.

Shifting momentarily back to the issue of access, who is really providing access today to the articles in the electronic versions of journals? Yes, the library still is, in the sense that viewing the full text of the articles in a given journal depends on the library having a paid subscription to the journal and on the viewer being authorized. ${ }^{17}$ But who is actually providing access, that is, where does control of the journal and its content reside? Remember what was said earlier about the organization and access the library provides via the catalog: access is more often than not provided via a hyperlink to the source or location of the journal. And this is by and large no longer with the library as it might be with a purchased copy of a given volume but, rather, with the publisher, who simply authorizes - or not-access to its own journal servers on which the content resides. The publisher provides the library with the appropriate links when a subscription has been started, and the library makes these links available to its patrons.

The question of ownership has been a sticky one with libraries and publishers since the advent of e-journals. Ownership is not an issue when a library has both a print and an electronic subscription; however, it is an issue when the only format being subscribed to is electronic. Then ownership is crucial, but the issue of preservation responsibility, tied handin-hand with ownership, becomes problematic and called into question. Despite the real source of access remaining with the publisher, ownership remains an important concept to the library. The reason is that if the library should ever have to cancel a subscription (which it must do with increasing frequency in these difficult financial times), it must have some guarantee of access to the years to which it subscribed, just as it would with print. With print, the question of continued access simply does not arise because the library has each volume and issue in 
hand that was purchased during the subscription years. So it still has a collection should it ever have to cancel. It is not so simple with the electronic format because the library never, or at least seldom, has "in hand" the electronic versions. These usually remain with the publishers. But libraries have been insisting on guaranteed rights of access to subscription years and have, on the whole, been successful in this endeavor. Many publishers have been agreeing to these terms so that libraries will either have "perpetual" access to their subscription years via the publisher's Web server or, in some cases, will be provided by the publisher with a CD-ROM containing the content to which the library had actually subscribed before cancellation. In the latter case, of course, the library would be responsible for mounting this content on its own internal servers for distribution and preservation, which raises the next issue.

The relationship between publisher and library probably has changed most in the area of preservation, and this is due to the nature of electronic publishing, which automatically entails providing the means for access. Access really is no longer through the library in the technical sense but, rather, through the publisher. And this process entails setting up elaborate systems of storage (again, formerly the responsibility of the library). In addition, many publishers such as ACS, IOP (Institute of Physics), AIP, and APS, to name but a few, have mounted large campaigns to digitize older content and make it available electronically as well, thus establishing vast archives (in the case of ACS, going back to 1887 for volume 1 of the Journal of the American Chemical Society). This is a wonderful thing to have because access now can be provided to historical content via the Web to the reader's desktop; however, creation of this "archived" content only cements the new responsibility that publishers have backed into, namely, keepers of the store of knowledge (at least to the degree they are willing to accept it).

The main concern of libraries, as it also should be for writers and readers alike, is that publishers have no history of this responsibility and there is no guarantee that they will fully appreciate the importance of the commitment to preservation. Again, that has simply not been their historical domain. This is a foreign concept, especially for commercial publishers whose interest has always been one of profit, which traditionally comes from the money made with new publications, not from the sale of historical publications. That has just not been their business. ${ }^{18}$

Libraries have made great strides recently, however, in getting publishers to recognize the enormous responsibility that is entailed when one takes on the task of ownership and access to the world's scholarly material. The society publishers, such as the ones mentioned above, have been good at acknowledging the importance of this matter and investing seriously in the technological infrastructure necessary to provide some assurance of longevity and even permanence of such ethereal collections. ${ }^{19}$

It was mentioned earlier that publishers are beginning to make electronic content available in a physical sense (CDROMs for mounting on internal servers), and this is actually providing a way for libraries to maintain some sort of control over preservation. In addition, many publishers are now actively working with an ever-increasing number of libraries in a program called LOCKSS (Lots of Copies Keeps Stuff Safe) ${ }^{20}$ This program allows participating institutions to create and maintain automatically digital "caches" of e-journal content from participating publishers, provided the caching library already subscribes to the content. Integ- 
rity of the collections is maintained by the LOCKSS software through periodic comparisons and verification of material from the various archiving institutions. As of this writing, almost a hundred libraries from all over the world are participating, with more than sixty publishers endorsing the program and providing content. This bodes well for the future of electronic preservation and provides another avenue for libraries to explore, thereby avoiding forced reliance on publishers who have no experience or track record in historical preservation of the written record. The cooperation of publishers with libraries in this initiative is most welcome, as this new format represents uncharted waters for both groups in the area of preservation. ${ }^{21}$

Although ownership and access already have been discussed within the scope of libraries, another aspect of this, which is quite important to the discussion at hand, has not yet been addressed. That aspect is the library's traditional role in providing patron access to articles that it does not own. In the context of the present discussion, what effect do changes in electronic writing and publishing within physics and chemistry have on this role of libraries? And what are the consequences of these changes to both writer and reader? There are hidden issues here as well that need to be uncovered.

A large part of a library's "collection" is the set of those items that it does not actually own either in print or have access to electronically. It is hard to think of this as part of a collection, yet the provision of this content is an enormous part of the library's responsibility to the research faculty and students it serves. No library can afford absolutely everything necessary to support the research needs of its patrons, especially in times when budgets are tight and subscriptions are dropping in great numbers. So a library negotiates borrow- ing arrangements with other libraries, institutions, or document delivery services to provide these documents, sometimes for free and often at a cost (certainly if the service is a commercial one). The role of interlibrary borrowing (ILB) and document delivery to a library in supplying patrons with needed articles has, in fact, become increasingly important. It has provided libraries with a mechanism for cutting expenses by canceling expensive and underused (that is, used too little to justify expense) journals and replacing them with ILB or other document delivery services to supply content from that journal. Despite severe budget cuts, the savings that can occur within this model often can not only enable the library to avoid financial disaster, but also free up enough funds to purchase new titles that might be needed. Just such a program was initiated successfully in 1993 by LSU libraries and undertaken again in 2004, the first instance later documented in two journal articles. ${ }^{22}$ Because of the increasing reliance by libraries on such ILB and document delivery programs to supply patrons with content, it is essential that the particular services used be able to maintain efficient delivery and highquality copies.

So how has the rapidly evolving nature of electronic writing and publishing affected this area of a library's responsibility to provide access to articles it does not own? It is precisely the two components mentioned above-speed of delivery and quality of copies - that are most affected by the changing nature of electronic writing and publishing in the sciences, particularly physics and chemistry. The following discussion looks at the aspect of speed first, with a bit of background highlighting the changes that have ensued and the reasons for them.

In the early 1990s, a document delivery service called UnCover came into exis- 
tence and promised a great improvement over the traditional ILB service, which consisted of borrowing or requesting article copies from participating institutions. Although there have been improvements in the quality of delivery, ILB service was - and still is, to a large degree - a laborious process taking one to two weeks to acquire a copy of an article. UnCover (later purchased by Ingenta) offered a much quicker alternative, featuring a stock of articles that could be faxed on request to paying subscribers to the service. Individual patrons of a subscribing institution could set up personal profiles that would include a fax number to which requested articles could be sent directly. Thus, if the desired article was available, the service offered an average twentyfour-hour delivery time directly to the patron-designated fax machine. This was a great improvement over the traditional system of ILB. Refined over a decade of use, the same service is offered today.

But speed of delivery remains an issue if the document delivery service is unable to fulfill a request, and there are several reasons why this might be the case. One reason is simply the fact that the document delivery service may not index or carry a particular journal's content. Another reason is an escalating copyright fee, courtesy of the publishers, but passed on to the subscribing institution by the third-party vendor, the document delivery service. This often causes the cost of an article to exceed the amount an institution is able to subsidize. And finally, publishers have been somewhat reluctant to allow their articles to be faxed by these third-party vendors.

And yet, we are seeing today a rapidly increasing need for speed for reasons that could not have existed before electronic publishing and are only now coming into play. This increased need for speed is a direct result of a new tendency by electronic publishers to publish papers online in advance of the print version. This certainly makes sense because the publisher can prepare an article to place online quicker than in print, so why not make it available to researchers as soon as possible? The problem becomes the quickly established dependency of researchers on these early editions, thereby leading to an inevitable divide between the haves and the have-nots. With competition among academic researchers becoming ever fiercer for grant dollars, prestige, and job security, those without electronic access to these early publications will be at a distinct disadvantage. A case in point is the Proceedings of the National Academy of Sciences (PNAS), a critical journal for a large segment of the sciences, particularly the biological sciences. Having a current print subscription is no longer sufficient. Through publication of PNAS's "Early Editions," a given paper may appear online up to seven weeks before a library will ever see it in print (this takes into account delivery time of the print version to the library), despite the fact that the library has a current subscription. What is required to keep one's faculty competitive in this case is nothing short of an electronic subscription. To be without this is to put an institution's faculty at a distinct disadvantage. So libraries are finding themselves forced to try to deal more and more with the publisher's electronic versions, if only for speed.

And yet there is another reason why libraries have to concern themselves directly with publisher versions. Even with the removal of obstacles preventing faxing, there remains another critical problem brought about by the ever-increasing capabilities within electronic writing and publishing in physics and chemistry. These new capabilities have to do with the quality of images, an aspect of physics and chemistry that has already 
been shown to be of great importance. With the ability of these subjects to produce increasingly higher-quality images in labs and with computer software, and the ability of publishers to reproduce those images electronically, has come the need for libraries and document delivery services to supply them in the quality needed by their readers. In other words, critical content in the details of the images must be transmitted or meaning is lost. Patrons have been finding the faxed copies of articles no longer to be of the quality necessary for them to extract all the content in the course of reading because of the inability of the fax process to transmit adequately the fine points of the images involved. Admittedly, this has improved with the advent of Ariel, a software/scanning system designed to allow libraries to scan and transmit articles back and forth electronically via computer. But we are still dealing with the conversion of a print document to electronic, with the resultant electronic delivery having, by design, to be printed out before it can be delivered to the patron. The system is better, but not ideal. Whether the system is sufficient, however, is certainly open to question.

So physics and chemistry researchers are finding more and more occasions when the publisher's original PDF version is necessary for satisfactory reading. Some publishers have been cooperating with third-party vendors such as Ingenta to allow delivery via this mechanism, which solves not only the speed problem, but also image quality. Unfortunately, not all publishers have agreed to this method. Many have set up mechanisms for single-article purchase and delivery from their own sites. This is good for the publisher, if such tight control over its content is an issue, and it is good for the independent researcher who wishes to purchase on his or her own. But this mechanism is not good for the traditional library/academic research setting, where the library provides faculty members with research informational needs at no cost to them. And that is a service faculty justifiably continue to demand. Publishers have been reluctant to set up institutional accounts so that libraries may at least subsidize these purchases for their patrons, but there are signs that may be changing now. However, even if publishers agree to this, there are management problems associated with a library having to set up agreements with each individual publisher, thereby negating the service previously rendered by a central management system such as that provided by a traditional document delivery vendor. Such a scenario would create a management nightmare with associated cost escalation, for one then would have to contract with document delivery companies and individual publishers.

So it is clear that changes in electronic writing within the scope of physics and chemistry have consequences for the distributor, even within the set of materials a library does not "own," but still must supply to its readers. This emerges with the issue of speedy delivery and quality of images contained within the articles, as both must be maintained in this era of high-resolution electronic images and the increasing need of faculty for "immediate" delivery of articles. What is at stake, once again, is that tenuous relationship between writer and reader-the cord is easily broken here.

\section{Images: Web Environment}

What are the new possibilities alluded to earlier, opened up by advances or changes occurring in electronic communication, particularly as they pertain to chemistry? Those possibilities apply primarily to images and manifest themselves within the medium of the Web. So far, with all the 
newfound abilities to write, transmit, and publish electronically, we are still dealing with what might be called flat, 2D filesimages, whether of words or structures, that are simply fixed, two-dimensional representations. One can look at the image, but that is all. Of course, ten years ago, one might have asked, "Well, what more should I want to do with it?" And that question precisely is being answered in a remarkable fashion today when looking to the Web as an independent medium for experimentation, a virtual laboratory, if you will. A number of programs have sprung up in recent years that are capable of producing so much more in an image. Some of the more important programs include RasMol, Protein Explorer, Isis Draw, ChemDraw Net, Chem 3D Net, Chime, and Chemscape Server, but this list is by no means comprehensive.

In fact, an image is no longer just an image. ${ }^{23}$ With a program such as RasMol, one can create colorful 3D images of molecular structures, of DNA that can be rotated, twisted and turned, components subtracted, and special views enabled so that the viewer is no longer just a passive recipient of a static image but, rather, an active participant in the reading and understanding of the structure so depicted. Because we can "see" more, the imagination is stirred to an even greater degree than otherwise and possibilities for understanding the ramifications of a given structure are greater than ever. In addition to RasMol, there are Chem 3D Net and Chime, which are browser plug-ins that enable 3D renderings of molecules. Programs such as ChemDraw and Isis Draw allow 2D drawing of structures in such a way as to allow for searching of databases for matches of these structures in order to identify compounds. In addition, one can do substructure searching, which is wonderful for exploring compounds that share a user-defined structural skeleton.
Unfortunately, there is an issue with browsers here. Rasmol, for example, is rather picky about the browsers under which it will function; it will work under some, but not others and certainly not all. This lack of compatibility with so many different browser versions helps illustrate one of the major problems in transferring such capabilities as are represented here to the more mainstream and traditional area of writing and publication.

\section{Traditional versus Experimental}

How, then, do these continually and rapidly evolving Web mechanisms affect writer, publisher, and distributor (that is, what traps lie in wait for the unsuspecting)? The positive benefits are easy to see and thus are not enumerated here, but the problems associated with rapid Web development as outlined above will help define the major problems this article has been looking at all along through each of the components of the triumvirate under analysis and their complex interactions and interdependence.

First, the Web. The question was raised earlier of whether the new possibilities opened up with relation to image production and representation might be available in the traditional form of the scholarly journal, and if not, why not? As has been seen, authors have a great number of new tools for image and structure production, new Web-based programs continually emerging that are adept at creating and manipulating structures. Publishers also have used the Web extensively in the creation and dissemination of their scholarly journals. But is there a full integration yet of these two components of the Web in scientific writing-experimentation and scholarly journal publishing? And, indeed, should there be?

Of the publishers discussed in this article, APS, AIP, and ACS offer services for publication of supplementary material in 
conjunction with the articles the supplemental material is designed to support. ${ }^{24}$ It is within this "supplemental material" domain that a bridge exists between the Web as experimental laboratory and the Web as scholarly journal publication medium. In the traditional publication process, files of the sort described above (dynamic files) are all inevitably rendered into static 2D files, while being converted to either jpg or gif image files. Not so, however, with the supplemental material publication; these files are left intact, so dynamic images remain dynamic.

Why this dichotomy between the traditional (though it is still hard to think of any Web publication as traditional) and the experimental? Why cannot or do not publishers take full advantage of the potential of the Web? Part of the cause of this chasm between Web journal publishing and Web publishing at large may lie in the more traditional model of publishing that is still in use, namely, print. With print editions of journals existing side by side with corresponding electronic ones, there is likely a need to maintain the same content in each version. And when one features dynamic as opposed to static images, the content has changed and so has the relationship between the reader and the content being read.

Another likely reason would be the need for permanence within journals being published, an aspect of which has already been discussed. This concern also is beginning to be reflected in the following statement by APS in its Physical Review Web Submission Guidelines: "While every effort is made to ensure that EPAPS material will always be available, it is not currently subject to the strict archival rules and regulations of the published article." ${ }^{25}$ To maintain a viable publication that readers can continue to access and read for years and even centuries down the road, a publisher must use software or means of rendering that itself will remain stable and usable. Yet, this very notion is absurd given the rate of change in computers, both hardware and software, and the rapid rate of obsolescence. Even considering the life of a given word-processing software, how often is it updated or revised? How long does it take before the new versions do not read the old versions anymore? Within historical time, merely the blink of an eye. Yet, we now must be concerned about this if we are to migrate so completely to electronic texts, and that is certainly where the sciences are rapidly headed. So publishers must act somewhat conservatively, staying with formats that are at least presently "universal" or universally acceptable.

The Web, on the other hand, represents an arena where rapid experimentation takes place, which is as it should be. There must be some area that can act as a laboratory for the experimental, and the fact that this is a public arena is all for the better. The Web provides a remarkable electronic evolutionary ground where change is so rapid that its occurrence can be seen. Competing programs continually pop up; some survive, others do not. But the survivors continue to evolve, only themselves perhaps eventually to die out, replaced in the electronic ecosystem by yet other, more dynamic forms. However, publishers cannot afford to operate at this accelerated level of change, nor should they given their new responsibility as keepers of the record. The stakes are far too high, and there is a great deal more to learn about electronic preservation over the long haul. These are basically new and uncharted waters, although much work is being done to find solutions to the problems presented by this migration. These are exciting times and troubling times as the nature of writing itself metamorphoses into an entirely different creature. 


\section{Conclusion}

Permanence, access - these issues have been brought to the fore in the guise of the Web and its turbulent activities. More accurately, what is highlighted is the possibility of the negation of these qualities in the electronic written word, for what is not permanent will also, at some point, no longer be accessible. And this gets to the heart of the matter-the preservation of that essential relationship between writer and reader, which is currently maintained through the intricate relationship between writer, publisher, and distributor. What affects one affects all, and that is precisely what this article has been trying to uncover.

Following is a list of some of the problems encountered thus far and how they relate to each component:

- numerous compatibility issues with various types of software used from both the writer's side and the publisher's side; the rapidly changing nature of commercial writing programs whose userfriendly aspects drive author demands, which in turn drive publisher changes, and what about the reader;

- the increased need for high-quality images, no longer supplied adequately by traditional ILB means; improvement in electronic production of images and subsequent publication thereof, forcing change in distributors, as libraries try to cope with mechanisms no longer adequate for delivery of such;

- the need for interactive images; compatibility issues again with means of delivery, affecting writers and publishers and libraries; question of stability, with advancement in programs occurring at inverse relationship to stability and compatibility over the long haul;

- ownership and its associated responsibility of preservation, the radical alteration of roles of publishers and libraries with regard to this, and the ramifications to the connection between writer and reader.

All of these issues ultimately affect that which most concerns us - the relationship between writer and reader. In every case, this relationship is threatened. The question always to be aware of is: Will readers continue to be able to read these articles, or images, years down the road? Will there be a written record a century from now of the scientific advances, the human advances being made at the present? Or will every new advancement in electronic writing be embraced without thinking because of the allure, the promise, and the immediate gratification that comes with the current ease of writing, publishing, delivery, and reading of electronic publications, oblivious to the potential perils? What is of concern here is not that these new advances are being embraced and used but, rather, that we may be embracing and using them unaware of the pitfalls. Recognizing and understanding the perils puts us in a position to control and guide the course of future developments in electronic writing, and that is our goal, not to regress or step backward but, rather, to move forward wisely with an eye always open to the future, for there lies our reader, waiting.

\section{Notes}

1. Pointers to Frequently Asked and Answered Questions comp.text.tex [accessed 11 April 2004]. Available online from http://tug.org/tex-ptr-faq.

2. A Discussion with Leslie Lamport [accessed 11 April 2004]. Available online from http:// dsonline.computer.org/0208/f/lam_print.htm.

3. "Publication charge" is a set amount charged per page to authors of published papers with APS. This fee and the corresponding discount offered by APS apply primarily to Physical Review Letters. The full waiver of publication charges applies to properly prepared electronic submissions 
in the Physical Review A-E journals.

4. Submission: Frequently Asked Questions, "When will you accept manuscripts prepared by other word processors than the obsolete TeX?" April 2001 [accessed 11 April 2004]. Available online from http://publish.aps.org/esubs/faq.html.

5. Physical Review Web Submission Guidelines. August 2003 [accessed 11 April 2004]. Available online from http://publish.aps.org/esubs/guidelines.html.

6. AIP Publishing Services, Peer X-Press [accessed 11 April 2004]. Available online from http://www.aip.org/publishing/services/pxp.html.

7. The ability to search for chemical information within the journal literature via structures is provided by special indexing and abstracting services such as the Chemical Abstracts Service Division of the American Chemical Society and Thomson ISI Web of Knowledge. Although such services play a special role in connecting the reader to the information, the author considers this role secondary to the primary relationships under discussion in this paper and so chooses to concentrate on these. A knowledge of the role of indexing and abstracting services in the dissemination of information is certainly important in a complete understanding of the way information is sought in the sciences, particularly chemistry, but this particular role would be best discussed in a separate article.

8. Hans-Dieter Barke and Temechegn Engida, "Structural Chemistry and Spatial Ability of Different Cultures," Chemistry Education: Research and Practice in Europe 2, no. 3 (2001): 227-39. Available online from http://www.uoi.gr/cerp/2001_October/pdf/06Barke.pdf.

9. Ibid., 227.

10. An orbital is defined as "a region in which an electron may be found in an atom or molecule." A Dictionary of Chemistry, 3rd ed. (New York: Oxford Univ. Pr., 1996).

11. Eric R. Scerri, "Editorial 4," Foundations of Chemistry 2, no. 1 (2000): 1-4. Available online from http://www.chem.ucla.edu/dept/Faculty/scerri/pdf/ed_4.pdf.

12. J. M. Zuo, M. Kim, M. O'Keeffe, and J. C. H. Spence, "Direct Observation of D-orbital Holes and Cu-Cu Bonding in Cu2O," Nature 401 (Sept. 2, 1999): 49-52.

13. Colin J.Humphreys, "Electrons Seen in Orbit," Nature 401 (Sept. 2, 1999): 21-22.

14. See appendix for complete list.

15. See appendix for complete list.

16. The author uses the more fundamental notion of symbol for "word" here, thus including images in the entire concept.

17. Authorized means someone who is allowed by the publisher through terms of the licensing agreement with the library subscribing to the journal to access and read the full-text content of that journal. Within the scope of this paper, this means faculty, staff, and students of the institution of which the subscribing library is part.

18. This is rapidly changing, however, as publishers begin producing digital archives of their historical collection (past publications) for sale or subscription. With the greater ease of access that comes with the digital collections, more and more scientists are beginning to use the older literature with much greater frequency, an interesting phenomenon.

19. However, the difficulties of doing this with the corresponding uncertainties are considerable and would be better discussed in an entirely separate article.

20. LOCKSS: Permanent Publishing on the Web. [Accessed 11 April 2004]. Available online from http://lockss.stanford.edu/.

21. Victoria A. Reich, "Lots of Copies Keep Stuff Safe as a Cooperative Archiving Solution for E-journals," Issues in Science and Technology Librarianship no. 36 (fall 2002). Available online from http://www.istl.org/02-fall/article1.html.

22. Stephen J. Bensman and Stanley J. Wilder, "Scientific and Technical Serials Holdings Optimization in an Inefficient Market: A LSU Serials Redesign Project Exercise," Library Resources $\mathcal{E}$ Technical Services 42, no. 3 (1998): 147-242.

Jane P. Kleiner and Charles A. Hamaker, “Libraries 2000: Transforming Libraries: Using Document Delivery, Needs Assessment, and Networked Resources," College and Research Libraries 58 (July 1997): 355-74. Available online from http://www.lib.lsu.edu/collserv/colldev/serials.html.

23. Although a cigar is sometimes still just a cigar.

24. APS, however, actually uses AIP's Electronic Physics Auxiliary Publication Service (EPAPS).

25. Physical Review Web Submission Guidelines. August 2003 [accessed 11 April 2004]. Available online from http://publish.aps.org/esubs/guidelines.html.

26. ACS Paragon System, Preparing Manuscripts Using TeX/LaTeX. [Accessed 11 April 2004]. Available online from https://paragon.acs.org/paragon/application?pageid=content\&mid=tex_instructions.html\&parentid=authorchecklist\&headername=Preparing\%20Manuscripts\%20Using $\%$ 20TeX/LaTeX. 


\section{APPENDIX}

\section{ACS: Acceptable Formats for Hardcopy Submissions to Most Journals}

ACS Paragon System, Preparation and Submission of Hardcopy Manuscripts [accessed 11 April 2004]. Available online from https://paragon.acs.org/paragon/application?pag eid=content\&mid=hardcopysubmission.html\&parentid=authorchecklist\&headernam e=Preparation $\% 20$ And $\% 20$ Submission $\% 20$ Of $\% 20$ Hardcopy $\% 20$ Manuscripts.

Macintosh:

- WordPerfect 3.5

- Microsoft Word, through Word 2001

- Framemaker, through version 6.0 (saved as MIF)

IBM and Compatibles:

- WordPerfect, through version 9

- Microsoft Word for Windows, through Word 2000

- Framemaker, through version 6.0 (saved as MIF) 1

TeX/LaTeX users are restricted to using LaTeX2a. 1

ACS provides templates for each of its journals in the following formats: for communications, MS Word 98 and 2001 for Macintosh, MW Word 2000 and 97 for Windows; for articles, only MS Word 98 for Macintosh. PC formats include MS Word 2002, 97, and Word Perfect 9 and 8.

\section{Paragon System: Acceptable Formats}

ACS Paragon System, Preferred Software [accessed 11 April 2004]. Available online from https://paragon.acs.org/paragon/application?pageid=content\&mid=preferredsoftware. html\&parentid=authorchecklist\&headername=Preferred $\% 20$ Software.

- Microsoft Word

- MSWord 7.0 for Windows 95 or higher

- MSWord 98 for Macintosh or higher

- WordPerfect

- PDF

- RTF

- TeX (including TeX tar and zip)

- LaTeX (including LaTeX tar and zip)

- FrameMaker

- PostScript

Although ACS states that plain TeX and REVteX are accepted, as noted above, elsewhere in its online documentation, ACS states that these versions are discouraged. In fact, it goes on to say that within this format, only manuscripts prepared under LaTeX2e will be used in journal production and even then authors must adhere to specific guidelines ACS provides for use of this program. ${ }^{26}$ 